\title{
La recherche au musée du quai Branly-Jacques
} Chirac

Maurice Godelier et Anne-Christine Taylor

\section{(2) OpenEdition \\ Journals}

Édition électronique

URL : http://journals.openedition.org/actesbranly/758

ISSN : 2105-2735

Éditeur

Musée du quai Branly Jacques Chirac

Référence électronique

Maurice Godelier et Anne-Christine Taylor, «La recherche au musée du quai Branly-Jacques Chirac », Les actes de colloques du musée du quai Branly Jacques Chirac [En ligne], 8 | 2017, mis en ligne le 12 juin 2017, consulté le 08 septembre 2020. URL : http://journals.openedition.org/actesbranly/758

Ce document a été généré automatiquement le 8 septembre 2020.

(c) Tous droits réservés 


\section{La recherche au musée du quai Branly-Jacques Chirac}

\section{Maurice Godelier et Anne-Christine Taylor}

\section{Anne-Christine Taylor}

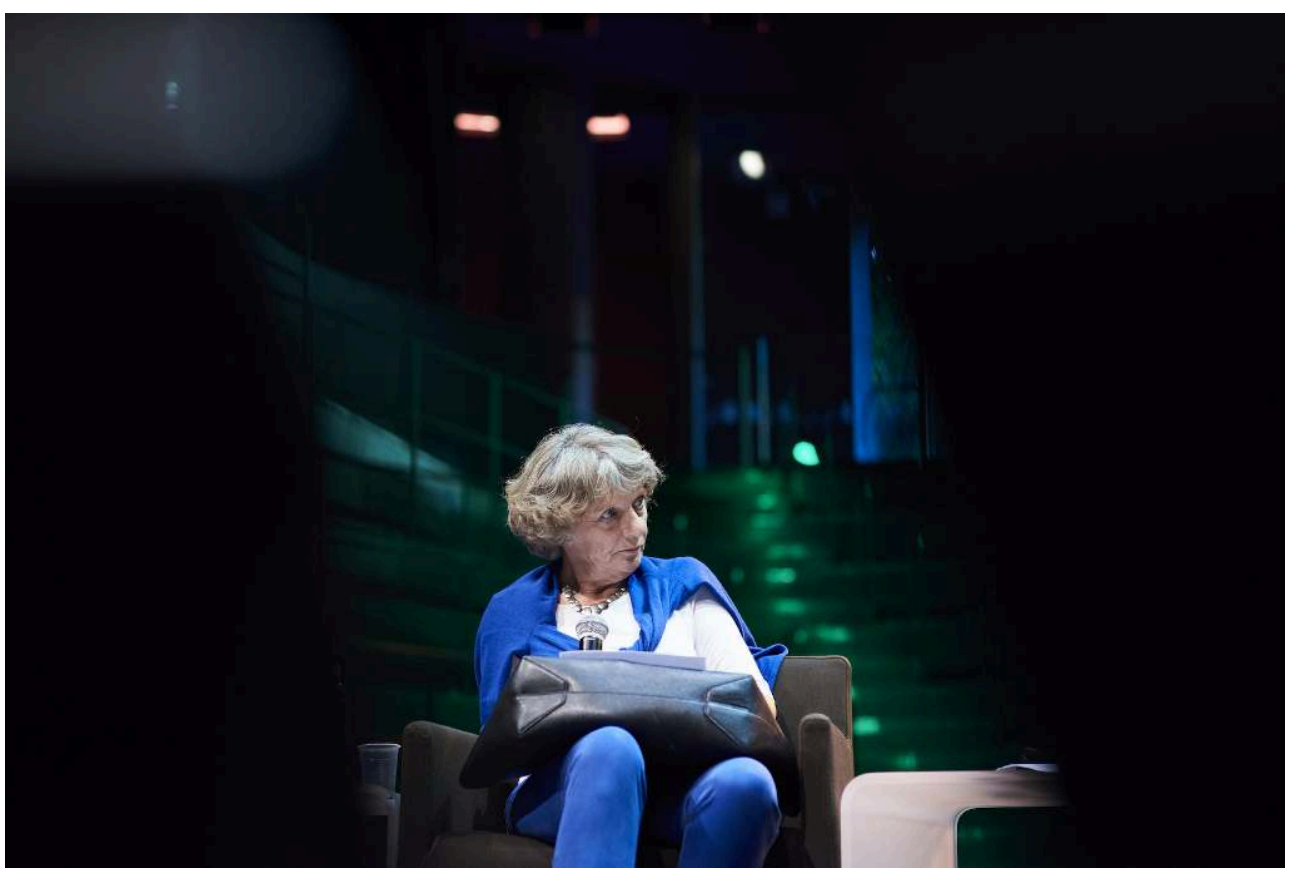

(C) musée du quai Branly - Jacques Chirac, photo Cyril Zannettacci

\section{Maurice GODELIER}

Une anecdote vous fera comprendre l'un des points de départ de cette histoire. Lionel Jospin m'avait demandé, quand il était Ministre de l'Education Nationale, de faire une visite d'évaluation du Musée de l'Homme et du Musée des Arts et Métiers. J'avais alors visité le Musée de l'Homme et j'avais constaté que dans certains départements, le département d'Océanie par exemple, on voyait des objets par terre, sans étiquette, sans 
indication de leur origine, etc. Lorsque j'ai rendu compte à Lionel Jospin de ma visite, je lui ai dit: " le musée a pris trop de retard. On ne peut plus le sauver. Il vaut mieux le fermer et jeter la clef». Lorsque Jacques Chirac est devenu Président, le projet de construire un nouveau musée faisait partie de ses propositions. Germain Viatte fut nommé Directeur de la muséologie du futur musée, mais les anthropologues dans leur majorité étaient hostiles au projet. Alain Juppé, alors Premier Ministre, n'avait pas encore attribué de budget pour le musée quand il fut obligé de démissionner. Lionel Jospin devint Premier Ministre et me fit demander par Claude Allègre ce que je pensais du projet de Jacques Chirac. Je lui répondis qu'il fallait y aller. Deux voix s'étaient élevées pour soutenir le projet. Celle de Lévi-Strauss et la mienne. Mais dès le départ, je prévins Lionel Jospin que le risque était de voir le nouveau musée se transformer en Musée d'Art et que les recherches sur les sociétés présentées dans les collections risquaient d'être sacrifiées ou fortement réduites. Jugez par vous-mêmes ce qu'il est.

2 Voilà un fragment de la préhistoire de ce musée. Je savais qu'il signifierait la disparition d'un musée ethnographique de type classique alignant sur des murs, dans des vitrines ou des tiroirs des centaines de flèches, de couteaux, d'objets d'origine et d'usage différents. De telles séries sont utiles pour le travail des chercheurs, mais elles n'ont pas nécessairement vocation à être exposées. Exposer c'est choisir car chacun sait qu'on ne peut pas présenter une société dans une ou plusieurs vitrines sans en mutiler la complexité.

3 Ce qui m'intéresse dans un musée c'est le nombre et la diversité culturelle, sociale, nationale de ses visiteurs. Quel professeur d'université, même très célèbre, pourrait rêver d'un million d'auditeurs par an à ses cours. Sur le net ce serait possible mais sans présence physique des personnes et des objets.

4 Mais les publics ne sont plus les mêmes parce que le monde n'est plus le même. Les empires coloniaux des pays occidentaux ont disparu et l'Occident doit prendre une distance critique par rapport à son passé et penser et faire voir autrement les objets accumulés dans ses musées pendant les siècles de son expansion et de sa domination mondiales. Une grande partie des objets du patrimoine de l'humanité se trouve en Occident. Une grande partie des objets d'Afrique se trouve en Europe et aux Etats-Unis. Nous sommes donc responsables de leur patrimoine qui est devenu nôtre. Nous le sommes vis-à-vis des Africains ou des Océaniens et vis-à-vis de nous-mêmes. Et nous nous trouvons au sein d'une France de plus en plus multiculturelle et visitée par des visiteurs provenant de pays de plus en plus nombreux et divers, au sein d'une France où se font jour de plus en plus des attitudes xénophobes. Donc un musée du XXI ${ }^{e}$ siècle se trouve investi de responsabilités politiques et culturelles nouvelles et fondamentales.

5 Dans ma vision d'un musée nouveau un principe pour moi s'imposait. Il fallait unir résolument les deux jouissances de l'art et du savoir. J'aime les objets à la fois pour leur beauté et pour leur sens. Mais un objet en général dit peu de chose sur lui-même audelà de sa matière et de sa forme. Un objet c'est de la matière qui a revêtu une forme symbolique et dont le sens obéit toujours à un code, à une intention et à un contexte. Restituer le sens qu'avait l'objet pour celui qui l'avait fabriqué implique de comprendre ce code et de découvrir l'intention et le contexte de sa production.

6 C'est au terme de telles recherches que l'on peut partager avec le public la connaissance de ce que voulaient faire ceux qui créaient pour eux-mêmes, pour leur propre usage, leur existence, les objets exposés dans nos vitrines. C'est là une tâche 
difficile et de longue haleine qui mobilise beaucoup de chercheurs de capacité et de sensibilité différentes et dont le travail doit se retrouver dans des banques de données mises à la disposition des visiteurs du musée. Mais ceci à condition que le public soit dans un environnement confortable pour jouir de ces connaissances à partager. J'avais la vision d'un musée enlaçant deux espaces. Un grand espace d'exposition où le public connaîtrait la jouissance de la découverte d'objets qui l'émeuvent et un espace d'interprétation où il pourrait revoir sur écrans les objets qui l'avaient ému et apprendre à les connaître. Connaître quoi ? D'abord à quoi servait l'objet pour ceux qui l'avaient fabriqué pour eux-mêmes et pour leur société ? Ensuite comment l'objet étaitil parvenu dans nos collections? Avait-il été acheté, par qui et à qui ? Ou volé, par qui et à qui ? Ou donné par qui, etc. Et qu'était la société d'où provenait l'objet à l'époque où l'objet l'avait quitté.

7 Tout ce travail de connaissance et d'information est très important. L'histoire des objets c'est l'histoire également des sociétés. C'est aussi notre histoire et celle des individus qui ont acquis et possédé ces objets. Ceci implique de travailler ensemble, anthropologues, conservateurs, historiens. Mais ceci implique aussi, s'il s'agit d'objets provenant du Mali, de travailler avec les Maliens. Comment les exclure puisque ces objets contiennent une partie de leur identité? L'époque coloniale est révolue. L'Occident ne peut plus se permettre de consommer pour son seul profit les richesses non seulement économiques mais culturelles de ses anciens empires. Soyons conscients du contexte dans lequel nous vivons et allons vivre. La Chine, l'Inde et d'autres pays veulent désormais continuer à se moderniser sans plus s'occidentaliser. Ils puisent dans leur passé qu'ils réinventent en partie pour se construire un avenir, une identité, sans l'Occident comme modèle. Pas sans échange avec l'Occident, mais sans l'Occident comme norme.

8 Un grand atout du musée du quai Branly est la large part qu'il fait aux expositions temporaires. De multiples aspects de la vie peuvent y être explorés tour à tour à l'aide d'objets qui en témoignent, les révèlent. Un objet, quel qu'il soit, un masque, une photo, un manuscrit est l'une des voies inventées par l'humanité pour transformer des idées et des rapports entre les hommes et avec l'univers qui les entoure en des matières et en des formes symboliques. Toute forme relève d'un code, connu, partagé par ceux qui ont créé l'objet avec cette forme. Nous essayons de retrouver ces codes et de les mettre à disposition du public. Mais, attention, jamais le public d'un musée ne verra les objets, ne les vivra comme ceux qui les ont inventés pour eux-mêmes. Il pourra en comprendre le sens originaire si on peut le lui expliquer mais il vivra l'objet des autres avec sa propre histoire, sa sensibilité, ses rêves.

9 En même temps la force des objets est que leur sens peut toujours échapper aux raisons, aux circonstances, à l'intention qui les ont fait naître. Et ceci précisément parce que leur forme et leur matière nous attirent ou nous repoussent et prennent alors de nouveaux sens. Une fois détaché de son origine, un objet commence une ou plusieurs nouvelles vies, celles que lui confèrent tous ceux qui le regardent, et éventuellement le touchent. Le regard disait Aristote est un " toucher à distance ».

Mais au cœur de toutes ces visions il existe les éléments invariants qui sont trop souvent ignorés de ceux qui conçoivent des expositions. Prenons l'exemple d'une très belle exposition: "La Mort n'en saura rien ». Ce qui manquait était de mettre en évidence le fait que dans toutes les sociétés et à toutes les époques la mort ne s'oppose pas à la vie mais à la naissance. Au-delà de la mort, l'individu commence une nouvelle 
vie. Une âme ou plusieurs âmes quittent le cadavre et vont continuer à vivre dans un lieu, un séjour qui leur est réservé. Cette idée, cette vision de la mort est contreintuitive. Mais elle est au fondement de toutes les religions, polythéistes et monothéistes. Que la mort ne soit pas la fin de la vie est un invariant commun à toutes les religions. Mais pour certaines d'entre elles s'ajoute un second invariant. Après la mort le défunt sera jugé pour ses actes au cours de sa vie. Et à l'issue de ce jugement post-mortem prononcé par des dieux ou par Dieu il retournera dans la roue des renaissances s'il est hindouiste ou bouddhiste ou il sera précipité en enfer ou admis au paradis.

11 Ne faut-il pas que ces choses essentielles à connaître soient présentes dans les expositions d'un musée car beaucoup d'objets en témoignent et l'humanité ne cessera jamais de rêver d'immortalité.

12 Un dernier point. J'ai vu ici des expositions magnifiques comme la dernière pour moi, celle sur le Sepik. Pourquoi, après chaque exposition, ne pourrait-on pas en faire figurer des éléments sur le net pour qu'elle se diffuse sur les plateformes de communication pour un peu plus de temps et pour plus de monde?

\section{Mme Anne-Christine TAYLOR}

Personne, à commencer par moi, ne contesterait les objectifs fixés par Maurice Godelier pour un musée tel que le nôtre, un musée du XXI ${ }^{e}$ siècle. Nous ne pouvons évidemment qu'y souscrire.

La question est plutôt à mon sens de savoir comment l'on s'y prend pour les réaliser. Il est évident que le musée ne peut pas à lui tout seul produire et rendre accessible au public le niveau d'information que Maurice Godelier envisage. Pour que le savoir irrigue ce musée et son public, il faut impérativement la collaboration active de chercheurs de tous les pays et de spécialistes extérieurs au musée. Il faut les intéresser à l'affaire. Or, c'est plus vite dit que fait. Je ne parle évidemment pas uniquement des remous, des controverses ou de la méfiance déclenchés par le projet du musée, mais d'un facteur plus structurel auquel Stéphane Martin a fait allusion ce matin.

Il faut tout de même rappeler que la question de la place de la recherche dans les musées avait, notamment au moment de la création de Branly, une forme très spécifique à la France. Ailleurs, dans les pays anglo-saxons, en Allemagne, dans les pays scandinaves, il va de soi que les professionnels de musées font et faisaient de la recherche. Il se trouve qu'en France, pour des raisons liées à l'histoire de l'institutionnalisation et de la professionnalisation des savoirs, la recherche quotidiennement pratiquée ou menée dans les musées était sortie du périmètre désigné par le label recherche, même dans les musées ethnographiques alors que même qu'à sa naissance l'ethnologie était étroitement associée aux institutions muséales. Corollairement, la recherche en sciences humaines, et en particulier l'ethnologie, s'était éloignée depuis plusieurs années du monde des objets dans leur matérialité. Le caractère nocif de cette séparation commençait à être clairement perçu depuis les années 1980. Mais lorsque l'on cherchait à y remédier, l'on continuait à imaginer qu'il suffisait en gros de greffer au musée un laboratoire de recherche «officiel» pour résoudre le problème. Or, il n'était que trop évident au moment de la création de Branly que cette formule-là ne marchait pas. Surmonter cette dérive des continents supposait donc plusieurs choses. D'abord, inventer de nouveaux dispositifs d'articulation avec le monde de la recherche en tournant le dos au modèle du laboratoire implanté dans le musée et en privilégiant au contraire des partenariats 
pluriels, flexibles, associés à des projets pluridisciplinaires à durée déterminée. Cela a effectivement été le sens de la création au musée d'abord d'un groupement de recherche international avec l'appui du CNRS. Plus tard, cette formule-là a été couplée avec l'entrée dans de grands programmes européens comme RIME ou aujourd'hui NEXUS, et la participation à ce que l'on appelle des Labex, c'est-à-dire ces programmes décennaux portés par des groupements d'établissements. Cela supposait aussi de faire du musée une zone d'attraction pour les étudiants et pas seulement pour ceux d'une seule école doctorale ou d'une seule université. Une des façons d'y parvenir est d'inviter le plus possible d'établissements à localiser des cours et des séminaires au musée du quai Branly. Enfin, cela impliquait aussi de convaincre la communauté académique internationale de la sincérité de l'intérêt que porte le musée aux pratiques de production de savoir, et notamment par la mise en place d'une très importante offre de bourses d'accueil pour de jeunes chercheurs de tous les pays, une offre très diversifiée et très ample au point qu'aujourd'hui le musée du quai Branly est probablement un des principaux réservoirs de bourses de recherche pour l'anthropologie et l'histoire des arts.

16 En sus d'inventer de nouveaux dispositifs pour faire entrer le monde de la recherche dans le musée et ouvrir celui-ci à la recherche pratiquée par les conservateurs, il fallait développer au musée des formes et des thèmes de recherche qui puissent intéresser et concerner à la fois les conservateurs et les académiques. Pour définir ce terrain-là, le mieux semblait de partir de ce que le musée était, à savoir non plus un musée d'ethnographie didactique à visée encyclopédique, mais un musée qui utilisait le prisme de l'esthétique pour introduire au thème de la diversité culturelle. Du coup, il paraissait cohérent de dresser notre étendard sur un territoire de recherche qui était en plus relativement peu développé en France, qui était celui de l'anthropologie et de l'histoire des arts au sens le plus large du terme - non pas «arts » au sens restrictif qu'il a dans l'usage ordinaire, mais « arts » au sens latin - et de centrer la politique de recherche du musée sur ce thème en le déclinant de multiples façons, jusque dans la revue Gradhiva produite par le musée. Il va de soi que dans cette déclinaison, l'histoire coloniale, l'histoire croisée, les formes non européennes de faire de l'histoire doivent occuper une place très importante. Le choix de ce domaine de recherche, celui de l'anthropologie et de l'histoire des pratiques de création, des pratiques des regards, de circulation, de réception des œuvres, permet de surmonter la division patrimoniale - totalement arbitraire et dépourvue de justification scientifique et encore moins politique - entre l'Europe et le reste du monde. De fait, l'on ne peut faire de l'anthropologie et de l'histoire des arts sans inclure l'Europe. D'ailleurs, l'Europe est souvent présente dans les expositions temporaires, même si le musée n'a évidemment pas de collection d'artefacts européens.

17 Comment assurer la meilleure diffusion du savoir au public en dehors des catalogues d'expositions ? Plutôt que d'imaginer un seul lieu de connaissance où le visiteur peut se documenter sur les objets du plateau ou d'une exposition, ne vaut-il pas mieux considérer que c'est l'ensemble du musée qui remplit cette fonction, en permettant un accès diversifié à divers niveaux d'information, partant des cartels minimaux (qui d'ailleurs ont été modifiés parce qu'ils étaient un peu trop minimaux au début) et des multimédias du plateau, en passant par les programmes multimédias didactiques maintenant implantés au salon Jacques Kerchache, les rencontres dans ce même salon, l'université populaire, les cours et les séminaires, les grands et les petits colloques, jusqu'à la bibliothèque de recherche du cinquième étage? Quant aux banques de 
données consultables sur le site du musée, elles répondent déjà en partie aux objectifs que Maurice Godelier a déclinés. Certes, la documentation ethnographique et historique des objets est encore d'ampleur et de qualité très inégale. Rappelons simplement qu'il y a largement plus de 300000 objets dans les collections et qu'il y a un peu moins de 15 conservateurs pour faire le constant travail d'actualisation de la documentation. C'est l'une des raisons qui ont motivé la création de bourses d'études des collections, pour permettre de contribuer à ce travail de documentation des collections. Ce travail de documentation se fait aussi, chaque fois que c'est possible, avec la collaboration de chercheurs issus des source communities. En ce moment même, une délégation de huit Indiens Wayanas des Guyanes est précisément en train de faire cela. Cela dit, lorsque l'on consulte la fiche d'un objet, l'on a maintenant enfin, en plus d'une fiche documentaire, d'emblée une bibliographie et un renvoi aux archives le concernant, ainsi qu'à d'autres objets du même genre; l'idéal étant que ces fiches ouvrent à terme directement sur des objets comparables dans les collections d'autres musées, par une mise en réseau de toutes leurs bases. Nous n'y sommes pas encore. Dans ce domaine-là comme dans d'autres, il est sûr que nous avons une marge de progression. Mais pour un paquebot comme celui-ci, et de façon générale pour des grands musées qui vivent dans un régime temporel très long, dix ans, c'est court. Avec le temps, nous finirons par nous approcher des objectifs que Maurice Godelier a mentionnés et avec lesquels tout le monde ici est d'accord.

\section{Maurice GODELIER}

Il y a une dizaine d'années, j'avais proposé à la Commission Européenne la stratégie suivante : inventorier tous les objets du Mali dans les musées européens et américains et reconstituer au niveau mondial une grande partie du patrimoine artistique et rituel des ethnies appartenant au Mali. Le Mali est un choix parmi de multiples autres possibles. Chaque objet est unique mais on en trouve de semblables dans plusieurs musées et peu à peu on peut remonter ainsi à l'artiste et au village où des objets de même type ont été créés. Les artistes, les créateurs africains sortiraient alors de l'ombre et de l'ignorance où ils furent pour la plupart d'entre eux depuis que leurs objets furent séparés d'eux. C'était le projet ECHO. Le projet recueillit des félicitations et fut enterré. Cette entreprise n'aurait pas été seulement française, mais européenne en étroite coopération avec les Maliens. Pourrait-on la ressusciter? En France des institutions telles le CNRS pourraient en être le support en coopération étroite avec les conservateurs des musées publics et privés.

\section{Mme Anne-Christine TAYLOR}

Effectivement, le projet Écho de mise en réseau de bases de données était en avance sur les moyens techniques disponibles à l'époque, ou en tout cas les moyens que le musée était disposé à y mettre. Il était encore coincé dans une approche par mots-clés. Il était littéralement insoluble de mettre en réseau des bases de données différentes à partir des mots-clés. Ce que permet maintenant de faire une architecture plutôt fondée sur des champs sémantiques. Cela change complètement la donne. C'est maintenant un horizon proche. On peut le faire et il faut le faire. 


\section{AUTEURS}

\section{MAURICE GODELIER}

Anthropologue, directeur d'études à l'EHESS, ancien directeur scientifique du musée du quai Branly-Jacques Chirac

\section{ANNE-CHRISTINE TAYLOR}

Anthropologue, directeur d'études au CNRS, ancienne directrice du département de la recherche et de l'enseignement du musée du quai Branly-Jacques Chirac 